OPEN ACCESS

Edited by:

Shikai Liu,

Ocean University of China,

China

Reviewed by:

Mehar S. Khatkar,

University of Sydney,

Australia

Xiaozhu Wang,

Auburn University,

United States

*Correspondence:

Ross D. Houston

ross.houston@roslin.ed.ac.uk

Diego Robledo

diego.robledo@roslin.ed.ac.uk

Specialty section:

This article was submitted to

Livestock Genomics,

a section of the journal

Frontiers in Genetics

Received: 27 November 2019 Accepted: 03 February 2020

Published: 27 February 2020

Citation:

Kriaridou C, Tsairidou S, Houston RD and Robledo D (2020) Genomic Prediction Using Low Density Marker Panels in Aquaculture: Performance Across Species, Traits, and Genotyping Platforms.

Front. Genet. 11:124.

doi: 10.3389/fgene.2020.00124

\section{Genomic Prediction Using Low Density Marker Panels in Aquaculture: Performance Across Species, Traits, and Genotyping Platforms}

\author{
Christina Kriaridou, Smaragda Tsairidou, Ross D. Houston * and Diego Robledo* \\ The Roslin Institute and Royal (Dick) School of Veterinary Studies, University of Edinburgh, Midlothian, United Kingdom
}

Genomic selection increases the rate of genetic gain in breeding programs, which results in significant cumulative improvements in commercially important traits such as disease resistance. Genomic selection currently relies on collecting genome-wide genotype data accross a large number of individuals, which requires substantial economic investment. However, global aquaculture production predominantly occurs in small and medium sized enterprises for whom this technology can be prohibitively expensive. For genomic selection to benefit these aquaculture sectors, more cost-efficient genotyping is necessary. In this study the utility of low and medium density SNP panels (ranging from 100 to 9,000 SNPs) to accurately predict breeding values was tested and compared in four aquaculture datasets with different characteristics (species, genome size, genotyping platform, family number and size, total population size, and target trait). The traits show heritabilities between $0.19-0.49$, and genomic prediction accuracies using the full density panel of $0.55-0.87$. A consistent pattern of genomic prediction accuracy was observed across species with little or no accuracy reduction until SNP density was reduced below 1,000 SNPs (prediction accuracies of $0.44-0.75$ ). Below this SNP density, heritability estimates and genomic prediction accuracies tended to be lower and more variable $193 \%$ of maximum accuracy achieved with 1,000 SNPs, 89\% with 500 SNPs, and $70 \%$ with 100 SNPs). A notable drop in accuracy was observed between 200 SNP panels $(0.44-0.75)$ and 100 SNP panels (0.39-0.66). Now that a multitude of studies have highlighted the benefits of genomic over pedigree-based prediction of breeding values in aquaculture species, the results of the current study highlight that these benefits can be achieved at lower SNP densities and at lower cost, raising the possibility of a broader application of genetic improvement in smaller and more fragmented aquaculture settings.

Keywords: breeding, disease resistance, growth, genomic best linear unbiased prediction (GBLUP), fish, oyster, salmon 


\section{BACKGROUND}

Aquaculture is the fastest growing food industry worldwide (Food and Agriculture Organization of the United Nations, 2018). While capture fisheries production has stagnated since the late $90 \mathrm{~s}$, aquaculture production has been consistently increasing $5.8 \%$ per year since 2001 (Food and Agriculture Organization of the United Nations, 2018), and this trend is expected to continue in the coming years to cope with the food demands of a growing human population. Nonetheless, aquaculture is still a relatively young industry, and although technological advances have been rapidly implemented to improve production volume and efficiency for some high-value species, these are slower in reaching the lowervalue, high-volume species that underpin most of global production. This is typified by genetic improvement technologies, where species such as Atlantic salmon have large and well-managed breeding programs akin to those for pigs and poultry, while most aquaculture species lag significantly behind. In part, this is due to the wide diversity of aquaculture species, with the top 20 animal species accounting for less than $80 \%$ of the total production (Food and Agriculture Organization of the United Nations, 2018) in contrast to terrestrial livestock, where four species are the source of $>90 \%$ of the world meat production. In addition, majority of aquaculture takes place in small to medium-sized farms, primarily situated in low to medium income countries. This context hinders the implementation of emerging technologies to help improve production, primarily due to their prohibitive cost.

One such technology is genomic selection, which utilizes genetic markers to identify the animals with the highest breeding values to select for producing the next generation (Meuwissen et al., 2001). Selective breeding programs are being increasingly utilized for aquculture species and have been shown to be highly effective in improving production traits, especially growth (Gjedrem and Rye, 2018). Genomic selection consistently outperforms family-based selection based on pedigree only (Zenger et al., 2018), leading to cumulative genetic gains over generations that incrementaly enhance the performance of farmed species. One of the main reasons underlying the slow uptake of genomic selection in aquaculture is genotyping cost. Genotyping usually relies on high-density SNP array platforms, which can be prohibitively expensive for routine application for most aquaculture breeding programs, due to the need to genotype thousands of performance tested fish (i.e. the reference population) and the selection candidates. One avenue to democratize genomic selection for smaller-scale, more fragemented aquaculture sectors is to exploit low-density SNP panels for which per-sample genotyping costs can be a fraction of the cost of SNP arrays.

However, it may be expected a priori that this cost-reduction due to reduced genotype density comes at the expense of reduced prediction accuracy in a breeding program. The improved prediction accuracy of genomic selection compared to pedigree-based approaches is primarily derived from an improved estimation of the genomic similarity between each pair of individuals. In most family-based aquaculture breeding programs, a procedure known as sib-testing (short for sibling testing) is performed whereby trait records are obtained from full siblings of the selection candidates - a process enabled by the high fecundity of aquaculture species. With pedigree-based selection, the genomic similarity between full-sibs is assumed to be $50 \%$, but the reality is that it can vary substantially around this value as a consequence of Mendelian sampling and linkage disequilibrium (Hill and Weir, 2011). In theory, the accuracy of estimating this genomic similarity should decrease as the density of genetic markers employed reduces, which would have a negative impact on prediction accuracy and consequently on genetic gain. However, in empirical studies of aquaculture species to date this decrease in accuracy seems to be relatively small and only observable once SNP densities drop to a few hundred markers [e.g. (Tsai et al., 2016; Correa et al., 2017; Gutierrez et al., 2018; Robledo et al., 2018; Vallejo et al., 2018; Yoshida et al., 2018a; Palaiokostas et al., 2019; Tsairidou et al., 2019)], which is likely a consequence of the large full sibling family sizes, such that long haplotypes are shared between many individuals in the reference and test population.

Therefore, low density genotyping appears to be a promising solution for enabling access to the benefits of genomic selection to a broader range of aquaculture species and sectors. However, the optimal SNP density to use is unclear and may be expected to vary depending on the species, population history, and trait of interest. The goal of this study was to assess if those variables affect the performance of low-density SNP panels and to determine if an optimal genotyping density can be identified as a practical, broad recommendation for aquaculture breeding programs. To do so, the performance of SNP panels of varying densities in estimating genetic parameters and breeding values was tested using previously published datasets for diverse aquaculture species, phenotyped for different traits, and genotyped with different platforms.

\section{MATERIALS AND METHODS}

\section{Datasets and Phenotypes}

Genotypes and phenotypes were obtained from four previously published studies in four different species, briefly: i) Atlantic salmon (Salmo salar) challenged with amoebic gill disease (AGD) were phenotyped for mean gill score (subjective 0-5 scoring system, commonly used as a measure of gill damage) and amoebic load (real-time PCR), and genotyped using a combined salmon-trout 17K SNP array (Robledo et al., 2018); ii) Common carp (Cyprinus carpio) were measured for growth traits (standard length and weight), and genotyped using RAD sequencing for $\sim 12 \mathrm{~K}$ SNPs (Palaiokostas et al., 2018); iii) Sea bream (Sparus aurata) challenged with Photobacterium damselae (causative agent of pasteurellosis) were measured for time to death, and genotyped using $2 \mathrm{~b}-\mathrm{RAD}$ sequencing for $\sim 12 \mathrm{~K} \mathrm{SNPs}$ (Palaiokostas et al., 2016); and iv) Pacific oyster (Crassostrea gigas) challenged with ostreid herpesvirus (OsHV-1- $\mu$ var) were measured for time to death and genotyped using a SNP array with $\sim 27 \mathrm{~K}$ informative Pacific oyster SNPs (Gutierrez et al., 2019). 


\section{Quality Control and Low Density SNP Panel Design}

Genotypes from the four datasets were filtered with PLINK v.1.9 (Purcell et al., 2007), excluding individuals with $>20 \%$ missing genotypes, and SNPs with $>10 \%$ missing genotypes, deviating significantly from Hardy-Weinberg ( $\mathrm{p}$-value $<10^{-6}$ ) and with minor allele frequencies $<0.05$. The number of SNPs excluded due to each QC threshold is shown in Supplementary Table 1. A summary of the genetic marker and trait data used for the four different datasets in this study after quality control is shown in Table 1. For reproducibility, QC-passing animal and SNP IDs are included in Supplementary Table 2.

SNP panels of varying densities were tested by taking subsets of the full QC-filtered SNP panel for each dataset. Panels of the following densities were tested in every species: 100, 200, 300, $400,500,600,700,800,900,1,000,1,200,1,400,1,600,1,800$, 2,000, 2,250, 2,500, 2,750, 3,000, 3,500, 4,000, 4,500 and 5,000. Additionally, 6,000, 7,000, and 9,000 SNP panels were tested depending on the total number of SNPs remaining after quality control (carp 6,000 SNPs; sea bream 7,000 SNPs; salmon and oyster 7,000 and 9,000 SNPs). The SNPs for each panel were selected using two different strategies (R package CVrepGPAcalc v1.0, https://github.com/SmaragdaT/CVrep/): i) random selection of SNPs within each chromosome (or linkage group for sea bream and oyster) where the number of SNPs selected from each chromosome/linkage group was proportional to its length; and ii) random selection of SNPs across the genome where SNPs were randomly chosen irrespective of their genomic position. For each SNP density, five different SNP panels were selected to account for potential bias arising from SNP subset selection. The results obtained with both selection strategies were similar, therefore only the results of the panels randomly selected across the genome are shown.

\section{Estimation of Genetic Parameters}

Heritabilities of the measured traits in each dataset were estimated using ASReml 3.0 (Gilmour et al., 2014) fitting the following linear mixed model:

$$
\mathrm{y}=\mu+\mathrm{Xb}+\mathrm{Za}+\mathrm{e}
$$

where $y$ is a vector of observed phenotypes, $\mu$ is the overall mean of phenotype records, $b$ is the vector of fixed effects, $a$ is a vector of additive genetic effects distributed as ${ }^{\sim} \mathrm{N}\left(0, \mathbf{G} \sigma_{\mathrm{a}}^{2}\right)$ where $\sigma_{\mathrm{a}}^{2}$ is

TABLE 1 | Summary of the datasets.

\begin{tabular}{|c|c|c|c|c|c|c|}
\hline \multirow{2}{*}{$\begin{array}{l}\text { Species } \\
\\
\text { Cyprinus } \\
\text { carpio }\end{array}$} & \multicolumn{2}{|c|}{$\begin{array}{l}\text { Individuals } \\
\text { before and } \\
\text { after QC }\end{array}$} & \multicolumn{2}{|c|}{$\begin{array}{l}\text { SNPs before } \\
\text { and after QC }\end{array}$} & \multirow{2}{*}{$\begin{array}{c}\begin{array}{c}\text { Full-sib } \\
\text { families }\end{array} \\
195\end{array}$} & \multirow{2}{*}{$\begin{array}{l}\text { Phenotypes } \\
\begin{array}{l}\text { Log length, } \\
\text { weight }\end{array}\end{array}$} \\
\hline & 1,214 & 1,211 & 12,311 & 6,966 & & \\
\hline $\begin{array}{l}\text { Crassostrea } \\
\text { gigas }\end{array}$ & 718 & 718 & 14,058 & 14,028 & 23 & Days to death \\
\hline Salmo salar & 1,481 & 1,481 & 16,582 & 9,866 & 85 & $\begin{array}{l}\text { Gill score, } \\
\text { amoebic load }\end{array}$ \\
\hline $\begin{array}{l}\text { Sparus } \\
\text { aurata }\end{array}$ & 777 & 741 & 12,085 & 7,598 & 73 & Days to death \\
\hline
\end{tabular}

the additive (genetic) variance and $\mathbf{G}$ is the genomic relationship matrix. $\mathbf{X}$ and $\mathbf{Z}$ are the corresponding incidence matrices for fixed and additive effects, respectively, and $\mathrm{e}$ is a vector of residuals. The identity-by-state genomic relationship matrix (G) was calculated using the GenABEL R package ["gkins" function; (Aulchenko et al., 2007)] kinship matrix (Amin et al., 2007), multiplied by two and inverted.

The different fixed effects included in the model for each species were i) tank (two levels) in Atlantic salmon, ii) factorialcross group (four levels) in carp, iii) none in sea bream, and iv) tank (two levels) in oyster.

\section{Genomic Prediction}

The accuracy of genomic prediction was estimated by ten replicates of fivefold cross-validation analysis (training set $80 \%$, validation set 20\%; $\mathrm{R}$ package CVrepGPAcalc v1.0, https:// github.com/SmaragdaT/CVrep). The phenotypes recorded in the validation population were masked, and genomic best linear unbiased prediction (GBLUP) was applied to predict the breeding values of the validation sets in ASReml 3.0, using the linear mixed model described above. Prediction accuracy was calculated as the correlation between the predicted EBVs of the validation set and the actual phenotypes divided by the square root of the heritability estimated from the full dataset $[\sim \mathrm{r}(\mathrm{y} 1$, y2) $\left./ \sqrt{h^{2}}\right]$.

\section{RESULTS}

\section{Trait Summary}

In total six traits were studied. Two traits related to Atlantic salmon resistance to AGD were used, gill score (subjective values $0-5$ ) and amoebic load (qPCR, Ct values), with means of $2.79 \pm$ 0.85 and $31.36 \pm 3.24$, respectively. The estimated genomic heritability values were moderate for both phenotypes, $0.22( \pm$ $0.04)$ for gill score and $0.24( \pm 0.04)$ for amoebic load. Two growth traits were studied in carp, length, and body weight, with means of $77.01 \pm 7.11 \mathrm{~mm}$ and $16.33 \pm 4.58 \mathrm{~g}$ respectively. Length showed a skewed distribution, deviating significantly from normality, and therefore was log-transformed. The heritability estimates were $0.27( \pm 0.04)$ for log-transformed length, and 0.19 ( \pm 0.04) for carp weight. Days to death were measured in pasteurellosis infected sea bream. The mean and standard deviation of surviving days for sea bream was $10.40 \pm$ 4.08 , and the heritability was $0.20( \pm 0.06)$. The same trait, days to death, was measured in oyster infected with OsHV-1- $\mu$ var. Survivors were assigned a value of eight for the variable "days to death". The mean for this trait was $6.76 \pm 1.91$ days, and the heritability $0.49( \pm 0.05)$.

\section{Reduced SNP Panel Densities Decrease the Precision of Genomic Heritability Estimates}

Heritabilities for the six traits were recalculated using the reduced density SNP panels (Figure 1). In general, decreasing marker density led to progressively lower heritability estimates; 


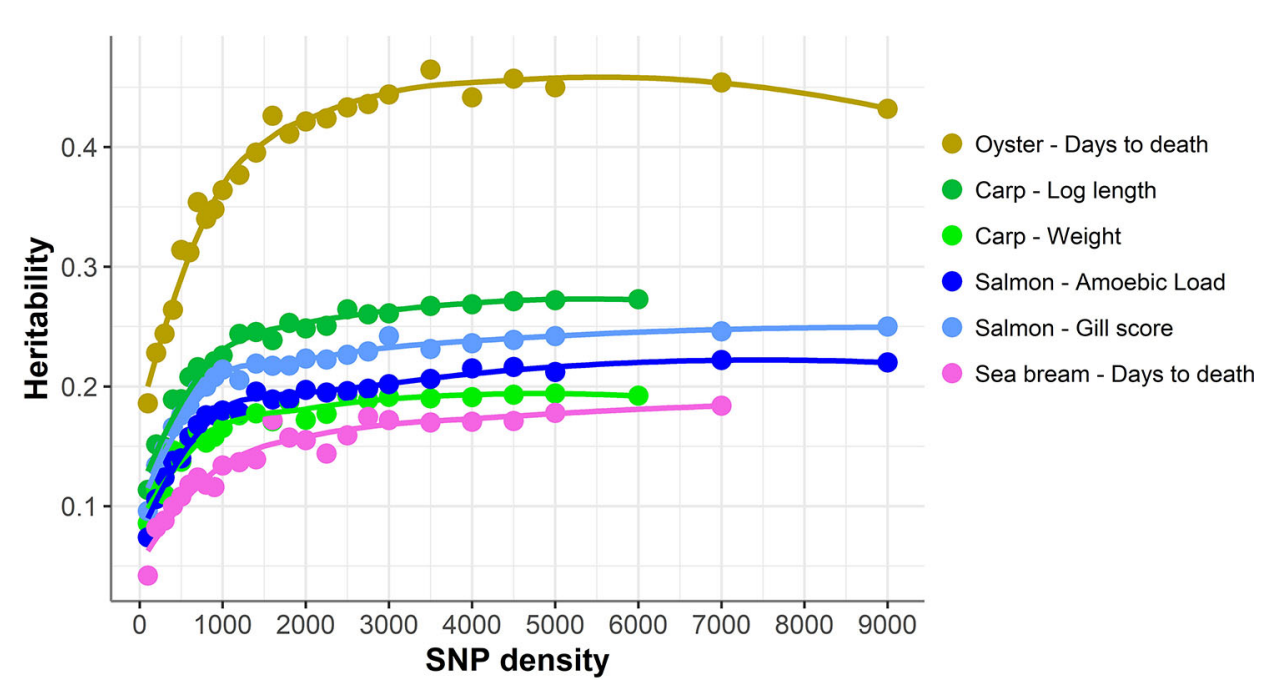

FIGURE 1 | Heritability estimates using low-density panels. The heritability was calculated using a linear mixed model with the genomic relationship matrix obtained with each low-density panel. For each density we used five different low-density panels, and the average of the heritabilities of the five panels is shown. The trend line was calculated using a Loess regression (local polynomial regression, span $=0.75$ ).

however, a clear downward trend is only observed when SNP density is reduced to $\sim 1000 \mathrm{bp}$ and below. The heritability estimates obtained for 100 SNPs decreased from 59 to $77 \%$ compared to the full density panel, while for 200 SNPs the decrease was on average $\sim 50 \%$.

\section{Genomic Prediction Using Reduced SNP Panels}

The accuracy of genomic prediction was evaluated using ten replicates of five-fold cross-validation (training set $80 \%$, validation set $20 \%$ ) for five different panels per SNP density (Figure 2). Since the heritabilities decrease substantially with lower panel densities, the accuracy of genomic prediction for all cross-validation analyses was calculated using the heritability obtained with the whole SNP panel, considered to be the most accurate heritability for the trait. Genomic prediction accuracy remained practically unchanged for every dataset until marker density was reduced below $\sim 2,000 \mathrm{SNPs}$, and a steep decrease was observed only for $\leq 1,000$ SNPs. The common trend observed accross the different species, traits, and genotyping platforms is clearly observed by plotting the proportion of the full SNP panel accuracy achieved with each low-density panel (Figure 3). Despite the significant differences between datasets and traits, the trend of the genomic prediction accuracies obtained with

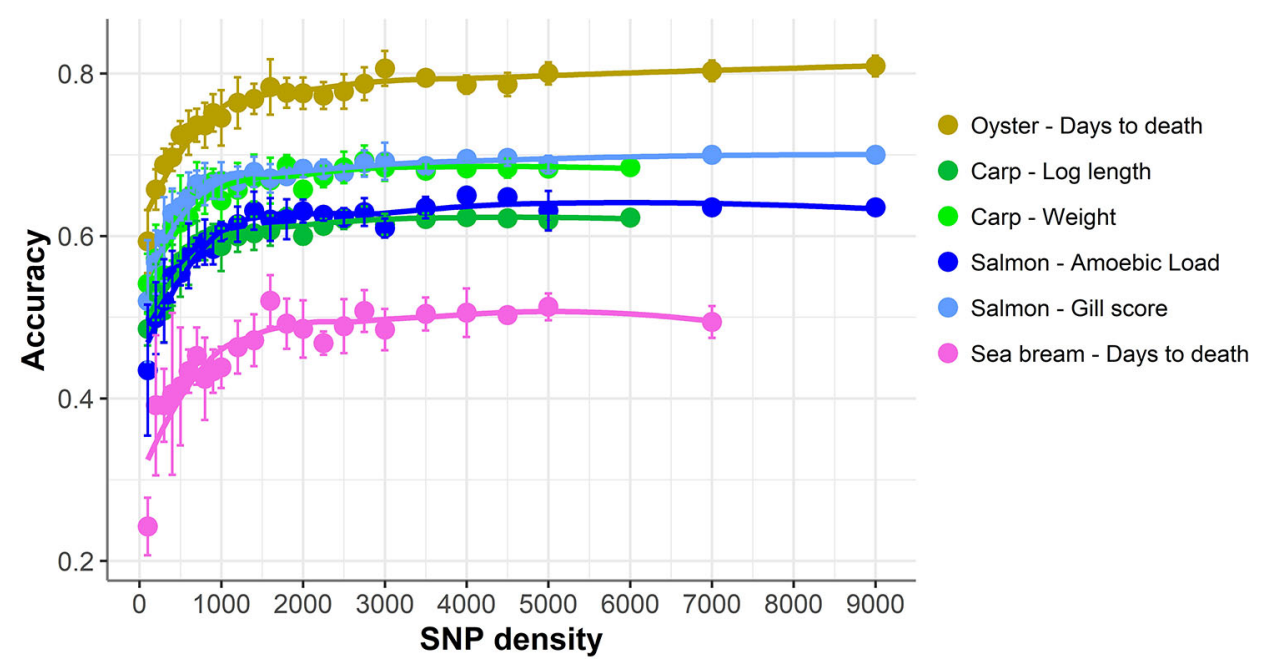

FIGURE 2 | Genomic prediction accuracy using low-density panels. Mean accuracy and standard deviation of genomic prediction for five different SNP panels per density. The trend line was calculated using Loess regression (local polynomial regression, span $=0.75$ ), and the shaded areas represent the confidence intervals. 


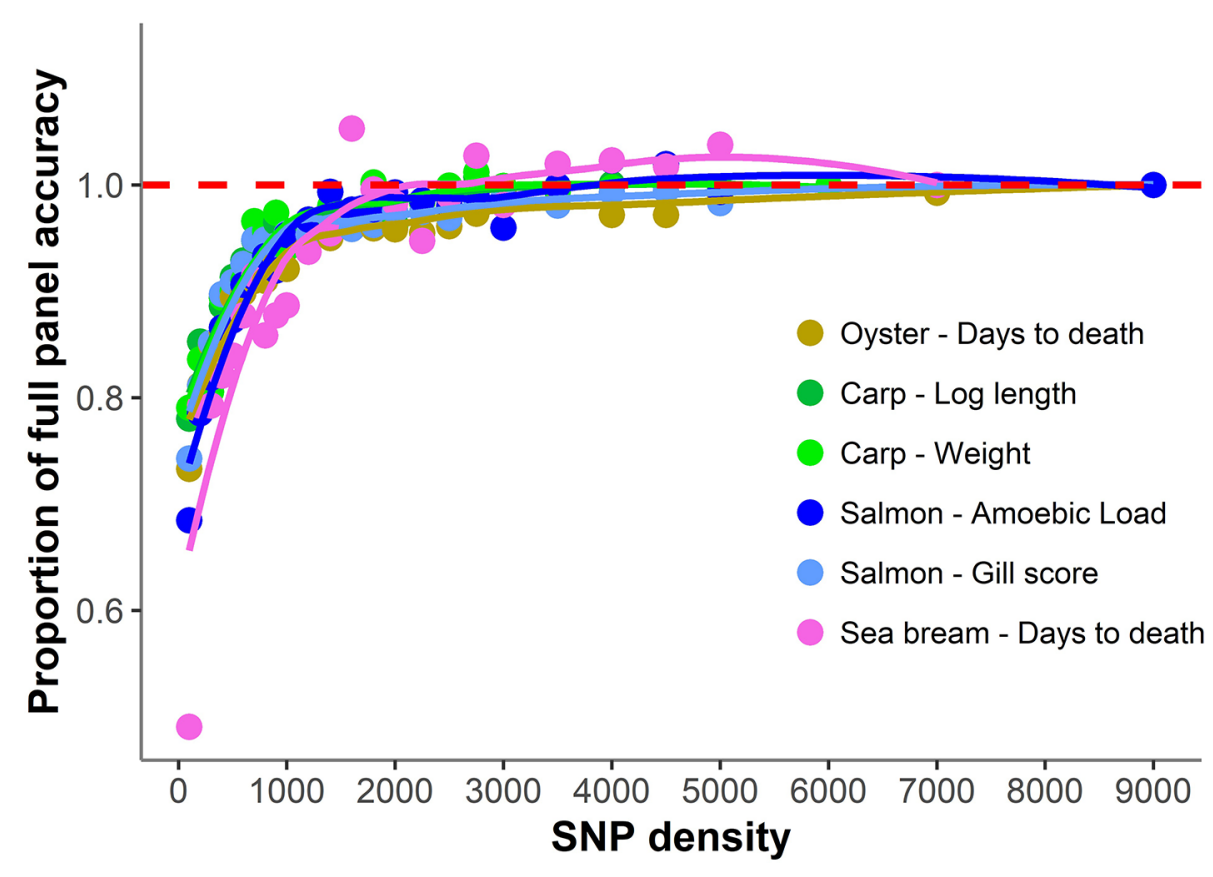

FIGURE 3 | Proportion of genomic prediction accuracy achieved with low-density panels. The proportion of accuracy achieved by each SNP density was calculated by dividing the mean accuracy at that density by the mean accuracy obtained using the full high density SNP panels. The trend line was calculated using a Loess regression (local polynomial regression, span $=0.75$ ).

low-density panels were remarkably similar. The average proportion of the full panel accuracy achieved with 2,000 SNPs was 0.97 , with 1,000 SNPs 0.93 , and with 500 SNPs 0.89 . With 100 SNPs the accuracy was reduced to 0.70 of that obtained with the whole density panel.

In addition, with decreasing SNP density the differences in prediction accuracy between different replicates of SNP panels of the same density increased (Figure 4). Therefore, SNP selection seems to be more relevant for the design of low-density panels than for higher density panels. On average, the difference between the maximum and minimum accuracies achieved by 100 density SNP panels was 0.11; salmon mean gill score showed the largest difference (0.19) and carp Log standard length the lowest (0.05).

\section{DISCUSSION}

Genomic selection has clear potential for improving prediction accuracy and genetic gain in aquaculture breeding programs, but the cost of genotyping can be prohibitive for many species and sectors. Therefore, since the price of per sample genotyping is generally associated with SNP density, knowledge of the lowest SNP density at which optimal genetic parameter estimation and genomic prediction can be performed is valuable. It may be expected that the optimal SNP density for genomic prediction would be species, traits, and genotyping platform-specific. In the current study, genotype and trait datasets from four diverse aquaculture species (Atlantic salmon, common carp, gilthead sea bream, and Pacific oyster), genotyped using different genotyping platforms (SNP array and RAD sequencing) were evaluated to search for common patterns of the impact of reducing SNP marker density on genomic prediction accuracy. The results were consistent across the different datasets, suggesting that a SNP panel between 1,000 and 2,000 SNPs would be sufficient for nearmaximal prediction accuracy for most polygenic traits in aquaculture populations. These results and their consistency are encouraging for lower-cost genotyping, and therefore improved affordability of genomic selection across different species and aquaculture sectors.

The uniformity of the results is relatively surprising considering the notable background differences between the four datasets. The trait, genotyping platform, family structure, population size or genome size seem to be relatively unimportant factors for the performance of low density SNP panels since genomic prediction accuracy trends were consistent across the four species. The large family sizes observed in most aquaculture species might partially explain these results. The genetic distance between training and validation populations has a large impact on the efficacy of genomic selection [prediction accuracy decreases with increasing genetic distance; (Scutari et al., 2016; Tsai et al., 2016; Tan et al., 2017; Palaiokostas et al., 2019)]. The underlying cause is that related individuals tend to share long haplotypes, which can be accurately captured with relatively sparse numbers of SNPs; however as genetic distance increases between training and validation, population haplotype length is reduced, and higher density panels are required to accurately capture the genomic similarity between animals. Most aquaculture species are highly fecund, and each pair of animals 


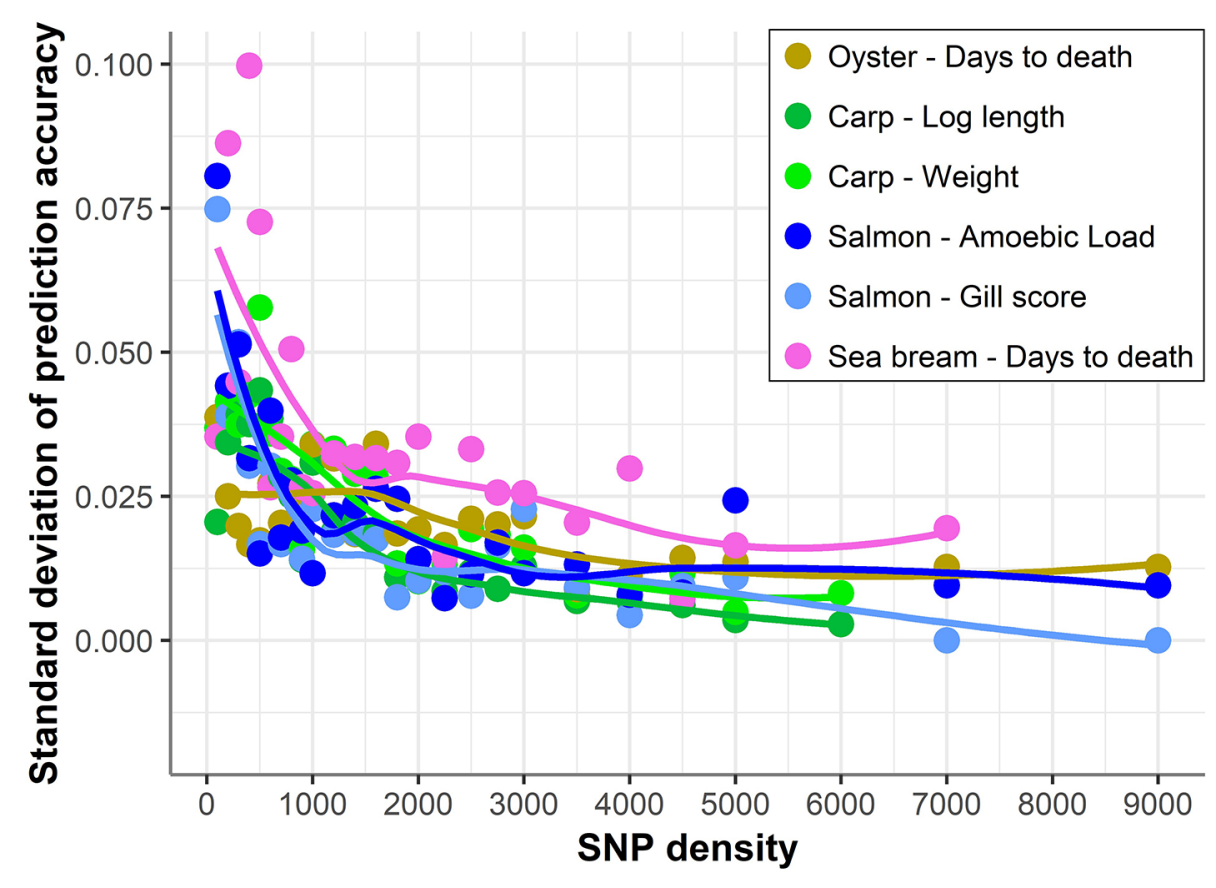

FIGURE 4 | Standard deviation of prediction accuracy using low-density panels. Variation in genomic prediction accuracy across the different SNP panels of the same density. The trend line was calculated using a Loess regression (local polynomial regression, span = 0.75 ).

frequently produces thousands of offspring, meaning that inclusion of multiple full and half siblings in training and validation sets is common practice. Consequently, although the accuracy of prediction might decrease in certain situations (i.e. cross-generational prediction to avoid the costs of generating new training populations), we consider that these results are generally applicable to polygenic traits in most aquaculture breeding schemes where close relatives of the selection candidates are routinely phenotyped.

Nonetheless, there will be situations where genomic prediction across generations or across populations is necessary. In these scenarios the shared haplotypes between pairs of individuals will be shorter, and therefore capturing genomic relatedness (if it exists; i.e. relatedness between unrelated populations will be zero and therefore of no use for prediction) is much more challenging and is likely to require higher SNP densities (Tsai et al., 2016). An avenue to increase the accuracy of low-density panels across sets of distantly related individuals could be the prioritization of variants that have a higher likelihood of directly effecting the trait in question rather than linked markers, for example, SNPs which fall in genes or other genomic features with a direct biological effect on the trait of interest and the utilization of selection models that exploit biological priors (MacLeod et al., 2016). However, establishing causal relations between genotypes and phenotypes is not trivial and will require extensive efforts in functional annotation of genomes [e.g. (Macqueen et al., 2017)] and collection of genotype and phenotype datasets across very large reference populations (Hickey, 2013). Consequently, low-density panels are not likely to be a feasible option for prediction across datasets without a high degree of relationship, which would require a large number of genome-wide distributed genetic markers. Nonetheless, this scenario is rare, and in the ample majority of aquaculture breeding programs full-sibs of the selection candidates are routinely phenotyped.

SNP panels consisting of $<1,000$ SNPs show a steep decline in genomic prediction accuracy, as does the estimated heritability, and the variation between replicate SNP panels of the same density increases. This suggests that low density panels are not accurately capturing the genetic relationship between animals, and that the performance of low-density SNP panels could be highly dependent on SNP choice. While leveraging additional layers of information might enable the design of high-performing low-density SNP panels, these would have to be tailored to specific breeding programs and might require substantial investment, i.e. an initial large-scale genotyping effort and extensive time commitment to determine the best panel, or potential functional experiments to establish marker function. Further, the performance of extreme low-density panels could fluctuate across generations as allelic frequencies vary. On the contrary, genotype imputation from very low-density panels (i.e. 100-200 SNPs) to medium density (i.e. $1 \mathrm{~K}-5 \mathrm{~K})$ might be a more generally applicable strategy to achieve the optimal balance between economic cost and genetic gain. Previous studies have shown the potential of imputation to achieve near-maximal accuracies in aquaculture populations (Tsai et al., 2017; Yoshida et al., 2018b); and a recent study by our group reported that imputation from 200 (offspring) to 5,000 SNPs 
(parents) results in selection accuracies similar to those obtained with $75 \mathrm{~K}$ SNP panels for sea lice resistance in Atlantic salmon (Tsairidou et al., 2019). In aquaculture, studies of imputation for genomic selection have been limited to salmonid species to date; however, it shows great potential and is likely to be a staple component of modern aquaculture breeding programs.

\section{CONCLUSIONS}

The patterns of loss of genomic prediction accuracy with reduced density SNP panels are strikingly consistent across datasets of different aquaculture species, despite their differences in population and family structure, phenotype and trait definition, and genotyping platform. These results suggest that SNP densities between 1,000 and 2,000 SNPs will frequently result in selection accuracies very similar to those obtained with high-density genotyping, irrespectively of the specifics of the breeding program design or population structure, assuming the presense of close relatives in the training and validation sets. Further, the higher variance between SNP panel replicates observed with decreasing density suggests that non-random SNP selection can increase the prediction accuracy of low-density panels. In summary, this study suggests that low-density SNP panels offer a cost-effective solution for broadening the impact of genomic selection in aquaculture, leading to improved enhanced performace of stocks and improved global food security.

\section{DATA AVAILABILITY STATEMENT}

All data used in this study has been previosuly published and is available in the corresponding article, namely Palaiokostas et al., 2016 (Sea bream), Palaiokostas et al., 2018 (Carp), Robledo et al., 2018 (Atlantic salmon), and Gutierrez et al., 2019 (Oyster).

\section{AUTHOR CONTRIBUTIONS}

$\mathrm{RH}$ and DR were responsible for the concept and design of this work. CK and ST designed and performed the genetic analyses.

\section{REFERENCES}

Amin, N., van Duijn, C. M., and Aulchenko, Y. S. (2007). A genomic background based method for association analysis in related individuals. PLoS One 2, e1274. doi: 10.1371/journal.pone.0001274

Aulchenko, Y. S., Ripke, S., Isaacs, A., and van Duijn, C. M. (2007). GenABEL: an $\mathrm{R}$ library for genome-wide association analysis. Bioinformatics 23, 1294-1296. doi: 10.1093/bioinformatics/btm108

Correa, K., Bangera, R., Figueroa, R., Lhorente, J. P., and Yáñez, J. M. (2017). The use of genomic information increases the accuracy of breeding value predictions for sea louse (Caligus rogercresseyi) resistance in Atlantic salmon (Salmo salar). Gen. Sel. Evol. 49, 15. doi: 10.1186/s12711-017-0291-8

Food and Agriculture Organization of the United Nations (2018). The State of World Fisheries and Aquaculture 2018. doi: 10.18356/8c28d3e2-en
CK, RH, and DR drafted the manuscript. All authors read and approved the final manuscript.

\section{FUNDING}

The authors gratefully acknowledge funding from the Scottish Aquaculture Innovation Centre (Grant number SL_2017_09) and BBSRC Institute Strategic Programme Grants to the Roslin Institute (BB/P013732/1, BB/P013740/1, BB/P013759/1). Christina Kriaridou was supported by Erasmus+ programme of the European Union (ER/SMP-OUT/2018-0033). The European Commission's support for the production of this publication does not constitute an endorsement of the contents, which reflect the views only of the authors, and the Commission cannot be held responsible for any use which may be made of the information contained therein.

\section{ACKNOWLEDGMENTS}

The authors acknowledge the contribution of Alastair Hamilton and Hendrix Genetics for generation of the Atlantic salmon data; Christos Palaiokostas, Martin Kocour and Martin Prchal for generation of the carp data; Christos Palaiokostas, Serena Ferraresso, Rafaella Franch and Luca Bargelloni for generation of the sea bream data; and Alejandro P. Gutiérrez, Jane Symonds, Nick King, Konstanze Steiner and Tim P. Bean for generation of the oyster data, which was funded by Cawthron's MBIE-funded Cultured Shellfish Programme, CAWX1315.

\section{SUPPLEMENTARY MATERIAL}

The Supplementary Material for this article can be found online at: https://www.frontiersin.org/articles/10.3389/fgene.2020. 00124/full\#supplementary-material

SUPPLEMENTARY TABLE 1 | Number of SNPs excluded because of different QC thresholds.

SUPPLEMENTARY TABLE 2 | Individual and SNP IDs passing quality controls for each dataset.

Gilmour, A. R., Gogel, B. J., Cullis, B. R. , and Thompson, R. (2014). ASReml User Guide, 4th Edn. (Hemel, Hempstead: VSN International Ltd).

Gjedrem, T., and Rye, M. (2018). Selection response in fish and shellfish: a review. Rev. Aquacult. 10, 168-179. doi: 10.1111/raq.12154

Gutierrez, A. P., Matika, O., Bean, T. P., and Houston, R. D. (2018). Genomic prediction for growth traits in Pacific oyster (Crassostrea gigas): potential of low-density marker panels for breeding value prediction. Front. Genet. 9, 391. doi: 10.3389/fgene.2018.00391

Gutierrez, A. P., Symonds, J., King, N., Steiner, K., Bean, T. P., and Houston, R. D. (2019). Potential of genomic selection for improvement of resistance to Ostreid Herpes virus in Pacific oyster (Crassostrea gigas). BioRxiv. doi: $10.1101 / 754473$

Hickey, J. M. (2013). Sequencing millions of animals for genomic selection 2.0. J. Anim. Breed Genet. 130, 331-332. doi: 10.1111/jbg.12054 
Hill, W. G., and Weir, B. S. (2011). Variation in actual relationship as a consequence of Mendelian sampling and linkage. Genet. Res. 93, 47-67. doi: $10.1017 /$ S0016672310000480

MacLeod, I. M., Bowman, P. J., Vander Jagt, C. J., Haile-Mariam, M., Kemper, K. E., Chamberlain, A. J., et al. (2016). Exploiting biological priors and sequence variants enhances QTL discovery and genomic prediction of complex traits. BMC Genomics 17, 144. doi: 10.1186/s12864-016-2443-6

Macqueen, D. J., Primmer, C. R., Houston, R. D., Nowak, B. F., Bernatchez, L., Bergseth, S., et al. (2017). Functional annotation of all salmonid genomes (FAASG): an internal initiative supporting future salmonid research, conservation and aquaculture. BMC Genomics 18, 484. doi: 10.1186/s12864-017-3862-8

Meuwissen, T. H., Hayes, B. J., and Goddard, M. E. (2001). Prediction of total genetic value using genome-wide dense marker maps. Genetics 157, 1819-1829.

Palaiokostas, C., Ferraresso, S., Franch, R., Houston, R. D., and Bargelloni, L. (2016). Genomic prediction of resistance to pasteurellosis in gilthead sea bream (Sparus aurata) using 2b-RAD sequencing. G3 6, 3693-3700. doi: 10.1534/ g3.116.035220

Palaiokostas, C. Robledo, D. Vesely, T. Prchal, M. Pokorova, D. Piackova, V., et al. (2018). Mapping and sequencing of a significant quantitative trait locus affecting resistance to koi herpesvirus in common carp. G3 8, 3507-3513. doi: 10.1534/g3.118.200593

Palaiokostas, C., Vesely, T., Kocour, M., Prchal, M., Pokorova, D., Piackova, V., et al. (2019). Optimizing genomic prediction of host resistance to koi herpesvirus disease in carp. Front. Genet. 10, 543. doi: 10.3389/fgene.2019.00543

Purcell, S., Neale, B., Todd-Brown, K., Thomas, L., Ferreira, M. A. R., Bender, D., et al. (2007). PLINK: a toolset for whole-genome association and populationbased linkage analysis. Am. J. Hum. Genet. 81, 559-575. doi: 10.1086/519795

Robledo, D., Matika, O., Hamilton, A., and Houston, R. D. (2018). Genome-wide association and genomic selection for resistance to amoebic gill disease in Atlantic salmon. G3 8, 1195-1203. doi: 10.1534/g3.118.200075

Scutari, M., Mackay, I., and Balding, D. (2016). Using genetic distance to infer the accuracy of genomic prediction. PloS Genet. 12, e1006288. doi: 10.1371/ journal.pgen.1006288

Tan, B., Grattapaglia, D., Martins, G. S., Ferreira, K. Z., Sundberg, B., and Ingvarsson, P. K. (2017). Evaluating the accuracy of genomic prediction of growth and wood traits in two Eucalyptus species and their F1 hybrids. BMC Plant Biol. 17, 110. doi: 10.1186/s12870-017-1059-6
Tsai, H. Y., Hamilton, A., Tinch, A. E., Guy, D. R., Bron, J. E., Taggart, J. B., et al. (2016). Genomic prediction of host resistance to sea lice in farmed Atlantic salmon populations. Gen. Sel. Evol. 48, 47. doi: 10.1186/s12711016-0226-9

Tsai, H. Y., Matika, O., Edwards, S. M., Antolín-Sánchez, R., Hamilton, A., Guy, D. R., et al. (2017). Genotype imputation to improve the cost-efficiency of genomic selection in farmed Atlantic salmon. G3 7, 1377-1383. doi: 10.1534/g3.117.040717

Tsairidou, S., Hamilton, A., Robledo, D., Bron, J. R., and Houston, R. D. (2019). Optimising low-cost genotyping and imputation strategies for genomic selection in Atlantic salmon. G3 10, 581-590. doi: 10.1534/g3.119.400800

Vallejo, R. L., Silva, R. M. O., Evenhuis, J. P., Gao, G., Liu, S., Parsons, J. E., et al. (2018). Accurate genomic predictions for BCWD resistance in rainbow trout are achieved using low-density SNP panels: evidence that long-range LD is a major contributing factor. J. Anim. Breed Genet. 135, 263-274. doi: 10.1111/ jbg. 12335

Yoshida, G. M., Bangera, R., Carvalheiro, R., Correa, K., Figueroa, R., Lhorente, J. P., et al. (2018a). Genomic prediction accuracy for resistance against Piscirickettsia salmonis in farmed rainbow trout. G3 8, 719-726. doi: 10.1534/g3.117.300499

Yoshida, G. M., Carvalheiro, R., Lhorente, J. P., Correa, K., Figueroa, R., Houston, R. D., et al. (2018b). Accuracy of genotype imputation and genomic predictions in a twogeneration farmed Atlantic salmon population using high-density and low-density SNP panels. Aquaculture 491, 147-154. doi: 10.1016/j.aquaculture.2018.03.004

Zenger, K. R., Khatkar, M. S., Jones, D. B., Khalilisamani, N., Jerry, D. R., and Raadsma, H. W. (2018). Genomic selection in aquaculture: application, limitations and opportunities with special reference to marine shrimp and pearl oysters. Front. Genet. 9, 693. doi: 10.3389/fgene.2018.00693

Conflict of Interest: The authors declare that the research was conducted in the absence of any commercial or financial relationships that could be construed as a potential conflict of interest.

Copyright (C) 2020 Kriaridou, Tsairidou, Houston and Robledo. This is an open-access article distributed under the terms of the Creative Commons Attribution License (CC $B Y$ ). The use, distribution or reproduction in other forums is permitted, provided the original author(s) and the copyright owner(s) are credited and that the original publication in this journal is cited, in accordance with accepted academic practice. No use, distribution or reproduction is permitted which does not comply with these terms. 